\title{
Correlation of the Cardiogenic Air Flow in the Respiratory Airway (i.e. the Pneumocardiogram) with Left Ventricular Stroke Volume in Dogs*
}

\author{
Jerry L. Wessale, Ph.D., Joe D. Bourland, Ph.D., \\ Charles F. Babbs, M.D., Ph.D., Rita C. Milewski, B.S.Pharm., \\ Michael E. Rockenhauser, B.S.E., \\ and Leslie A. Geddes, M.E., Ph.D.
}

\section{SUMMARY}

The pneumocardiogram (PNCG) is a record of the pulsatile flow of air in the trachea coincident with each heart beat. In 5 anesthetized mongrel dogs, the cardiogenic air flow through an endotracheal tube was measured with a research pneumotachograph. The correlation between the pneumocardiographic volume of air, obtained by integrating the PNCG over the period of ventricular ejection, and stroke volume (SV), measured by the saline-dilution method, was determined for a physiologic range of SV. Although the pneumocardiographic volume tracked measured SV, the former was always less. The range of correlation coefficients was 0.60 to 0.91 . The results suggest that the PNCG may be suitable for continuous tracking of changes in $\mathrm{SV}$ on a noninvasive, beat-by-beat basis. The technique is ideally applicable to intubated human subjects, particularly those undergoing closed-chest surgical procedures.

\section{Additional Indexing Words:}

Pneumocardiogram

Pneumotachograph

$\mathrm{F}$

OR over a century, physiologists ${ }^{1-3)}$ have recognized a cardiac-induced flow of air in the respiratory tract. This phenomenon is well known to anesthesiologists who frequently observe small pulsations-independent of patient breathing - in the valves of a closed-circuit anesthesia system. A record of the pulsatile flow of air in the trachea, coincident with each heart beat, is known as a pneumocardiogram (PNCG). This small, yet measurable

From the Biomedical Engineering Center, Purdue University, West Lafayette, IN 47907, U.S.A.

* Supported in part by grant HL22321 of the National Institutes of Health, Bethesda, MD.

Address for reprint: Jerry L. Wessale, Ph.D., Biomedical Engineering Center, Room 204, A. A. Potter Bldg., Purdue University, West Lafayette, IN 47907, U.S.A.

Received for publication October 23, 1984. 
flow of air is the basis of a simple, inexpensive and noninvasive index of cardiac stroke volume. Easily obtained during anesthesia, the PNCG may serve as a valuable adjunct to the usual clinical signs employed to assess a patient's status.

The PNCG has been investigated only slightly as a noninvasive indicator of cardiac status. To date, the relationship between ventricular stroke volume and the volume of air that moves into the lungs during ventricular ejection has not been quantified. Therefore, the objective of this study was to determine how well the systolic volume of air entering the trachea during ventricular ejection (calculated from the PNGG) tracks measured cardiac stroke volume in the dog during steady-state conditions.

\section{Materials and Methods}

\section{Animal preparation:}

Five mongrel dogs weighing from 11.0 to $18.0 \mathrm{Kg}$ were anesthetized with sodium pentobarbital (Diabutal ; Diamond Laboratories, Inc.; Des Moines, IA; $30 \mathrm{mg} / \mathrm{Kg}$, i.v.). Supplemental doses were given to maintain a stable depth of anesthesia. Each animal was intubated to provide a patent airway; the endotracheal tube was advanced so that its tip was located between the larynx and the bifurcation of the trachea. The animal was then placed in dorsal recumbency on a V-shaped animal board for the remainder of the study. A femoral vein was catheterized for administration of drugs and fluids.

The ECG (lead II) and aortic arch pressure were recorded continuously. Blood pressure was recorded via a fluid-filled, \#8-French pigtail catheter (Cook, Inc.; Bloomington, IN), connected to a strain-gauge pressure transducer (Model P23Db; Gould Inc., Instrument Division; Cleveland, $\mathrm{OH}$ ). The pressure catheter was introduced into a femoral artery and advanced retrograde through the aorta up to the arch.

Determination of stroke volume:

Cardiac output was determined by the saline-dilution method $^{4)}$ during steady-state conditions. To allow multiple and frequent determinations, a flow-through conductivity cell ${ }^{51}$ was placed in an arteriovenous shunt consisting of intravascular catheters previously inserted into a femoral artery and a femoral vein. A third catheter, for injecting the saline indicator, was inserted into the right atrium via the right external jugular vein. Heparin $(2 \mathrm{mg} / \mathrm{Kg})$ was given to prevent clot formation in the catheters and the conductivity cell. The $50-\mathrm{KHz}$ impedance of arterial blood between the electrode terminals of the flow-through conductivity cell was monitored contin- 
uously with an impedance recorder (Narco Bio-Systems; Houston, TX), which was in turn connected to a graphic recorder $\left(\mathrm{Physiograph}^{\mathrm{R}}\right.$; Narco Bio-Systems; Houston, TX).

A dilution curve was inscribed following the rapid injection of $2 \mathrm{ml}$ of $5 \%$ saline via the jugular catheter. Each dilution curve was calibrated electrically by momentarily short-circuiting a 5 -ohm resistor in series with the conductivity cell. ${ }^{4}$ This enabled the change in impedance of the blood to be expressed in terms of the change in concentration of sodium chloride in the blood. A small sample of arterial blood was then withdrawn to determine the packed-cell volume, which was needed to determine the concentration equivalent of the 5-ohm calibrating signal.4)

Dilution curves were recorded on a magnetic-tape recorder (Model Store 4D; Lockheed Electronics; Plainfield, NJ) and later displayed on a graphic recorder (Model 2600; Gould Inc.; Cleveland, OH). The graphic record of a dilution curve was digitized using a Graf/Pen ${ }^{\mathrm{R}}$ manual ultrasonic digitizer (Model NT-301; Science Accessories Corporation; Southport, CN) in conjunction with a PDP $11 / 03 \mathrm{G}$ laboratory digital computer (Digital Equipment Corporation; Maynard, MA). The dilution-curve area was determined by the method of Kinsman et al. ${ }^{6)}$ Cardiac output was then calculated according to the Stewart-Hamilton equation and stroke volume (in ml) was calculated by dividing cardiac output by heart rate.

Measurement of the cardiogenic air flow:

The PNCG was recorded using a research pneumotachograph described previously, ${ }^{\text {n-9) }}$ Briefly, a Fleisch \#2 air resistor was connected to a highsensitivity, wide-bandwidth differential pressure transducer, the output of which constituted the PNCG. Analysis of the PNCG during the post-expiratory pause of spontaneous breathing was possible when the respiratory rate was less than $20 / \mathrm{min}$. If, however, the rate exceeded 20 breaths $/ \mathrm{min}$, temporary apnea was induced and the PNCG recorded subsequently. Apnea was induced either by a brief period of hyperventilation or by intravenous administration of the neuromuscular blocker, succinylcholine. During longer periods of apnea, positive-pressure ventilation was maintained by a mechanical ventilator (Model 613; Harvard Apparatus Co., Inc., Millis, MA).

In all animals the volume of air moved in the trachea was calculated by integrating the PNCG over the period of left ventricular ejection, as illustrated in Fig. 1 for a typical PNCG. The period of ventricular ejection (the vertical dashed lines) was determined from the aortic pressure recording. The volume of air (the shaded area) was derived from a graphic record. The PNCG was recorded and digitized in the same manner as for the dilution 

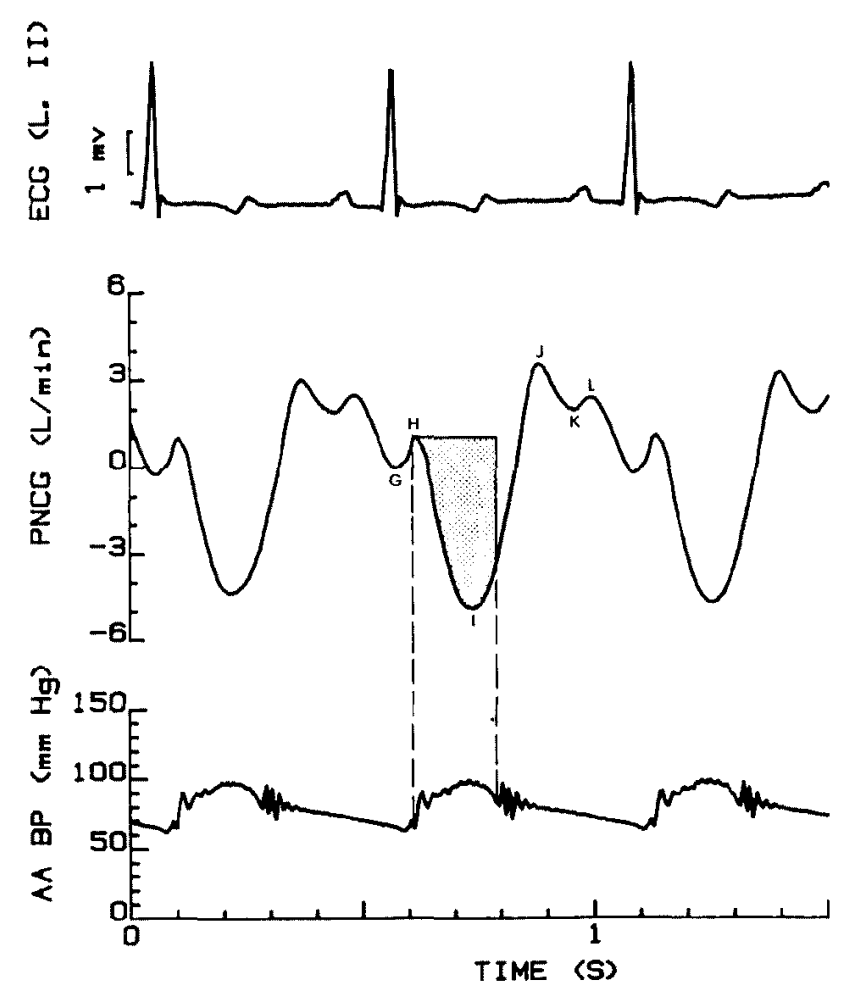

Fig. 1. Determination of the volume of air associated with ventricular ejection from the pneumocardiogram (PNCG). The period of ventricular ejection (dashed lines) is derived from ascending aortic pressure (AA BP). The shaded area represents volume of air.

curves. The volume of air represented by the PNCG was obtained by averaging the values for 3 to 4 selected beats occurring during inscription of a dilution curve.

\section{Experimental protocol:}

A PNCG-stroke volume determination consisted of 1) connecting the pneumotachograph directly to the endotracheal tube, 2) injecting the saline indicator and inscribing a dilution curve and 3) disconnecting the air resistor and subsequently ventilating the animal if spontaneous breathing was absent. Heart rate was constant and blood pressure was stable during inscription of the dilution curves. Stroke volume was altered over a physiologic range by several methods: 1) right atrial pacing, 2) vagal stimulation, 3) blood volume augmentation using canine blood or lactated Ringer's solution, 4) blood volume reduction by controlled hemorrhage, and 5) infusion of isoproterenol alone or in combination with atrial pacing or vagal stimulation. Not all manipulations were used in all dogs. 


\section{Results}

A typical pneumocardiogram (PNCG) is shown in Fig. 1. Air flow out of the trachea is indicated by an upward deflection; air flow into the trachea is indicated by a downward deflection. We have adopted the ballistocardiographic system of nomenclature (letters $G$ through L in Fig. 1) to designate the peaks and valleys in the PNCG; the same designations have been used in previous investigations. ${ }^{7,9)-11)}$ In particular, the $G$ wave is associated with atrial contraction, the large H-I-J component is associated with ventricular ejection, and the $\mathrm{K}$ and $\mathrm{L}$ waves occur during the period of ventricular filling. It should be noted that air moves into the lungs during the greater part of the ventricular ejection period. However, under steady-state conditions over one cardiac cycle, the net volume of air moved into the lungs by cardiac activity should be zero.

Figs. 2 and 3 present the pneumocardiographic volume plotted against measured stroke volume for $\operatorname{dogs} \# 1$ and \#2, respectively. The smallest regression coefficient was observed for dog \#1; one of the largest regression coefficients was observed for dog \#2. Table I summarizes the linear regression analysis ${ }^{12}$ ) of the data obtained from the 5 animals. The last column of Table I lists the range of heart rates observed, as well as the corresponding

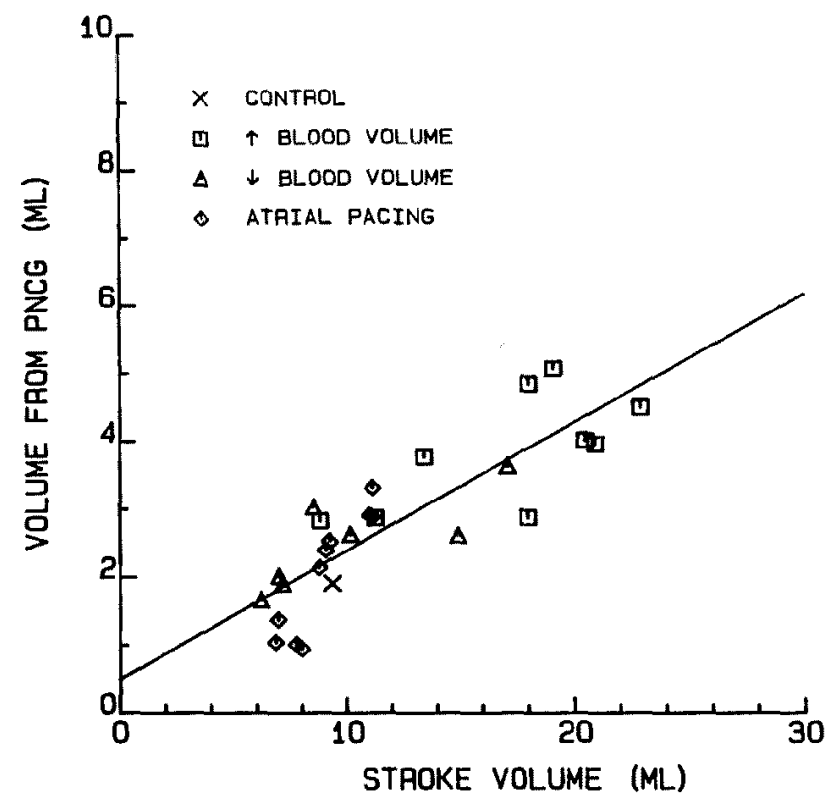

Fig. 2. Volume of air from PNCG versus stroke volume determined by the saline-dilution method for dog \#1. The least-squares line is represented by a solid line. 
means and standard deviations. The correlation coefficients for the data from each of the 5 experiments were statistically significant $(p=0.01)$. The range of correlation coefficients was from 0.60 to 0.91 . In general, the volume of air that moved into the trachea during ventricular ejection increased with increasing stroke volume. Although the pneumocardiographic volume

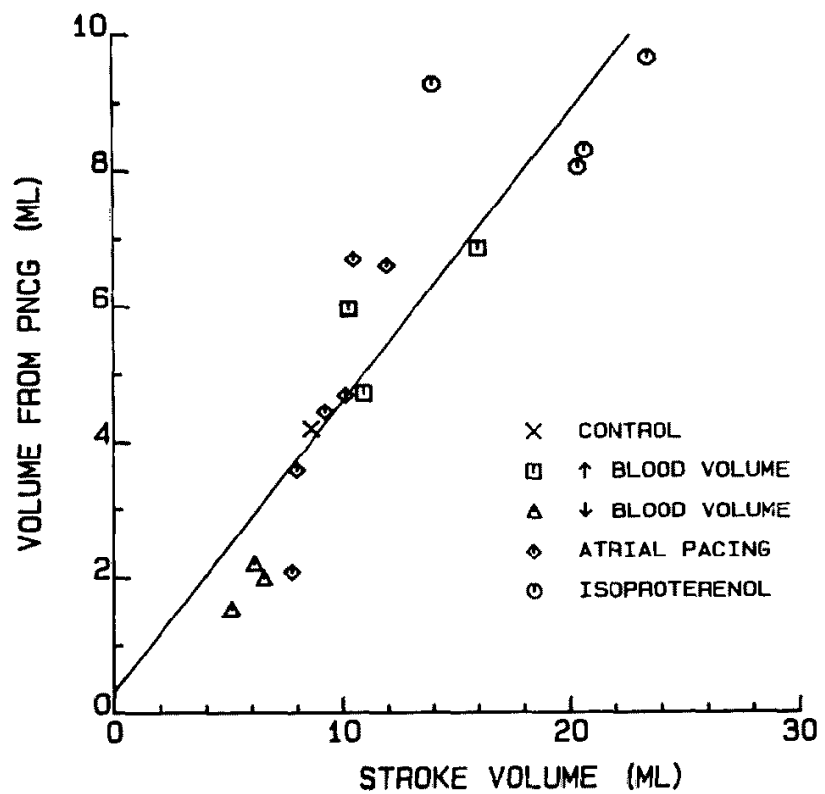

Fig. 3. Volume of air from PNCG versus stroke volume determined by the saline-dilution method for dog \#2. The least-squares line is represented by a solid line.

Table I. Linear Regression of Air Volume ( $y$, in $\mathrm{ml}$ ), Calculated from the PNCG, Versus Cardiac Stroke Volume ( $x$, in $\mathrm{ml})^{\mathrm{a}}$

\begin{tabular}{|c|c|c|c|c|c|c|c|c|}
\hline $\begin{array}{l}\text { Dog } \\
\text { ID }\end{array}$ & $\begin{array}{c}\text { Weight } \\
\text { (Kg) }\end{array}$ & n & $b_{1}$ & $\mathrm{~b}_{0}$ & $r$ & $\mathrm{~s}$ & $t$ & $\begin{array}{c}\text { Heart Rate } \\
(/ \text { min })\end{array}$ \\
\hline 1 & 11.0 & 27 & 0.19 & 0.50 & 0.84 & 0.615 & 7.88 & $\begin{array}{l}62-188 \\
120.5 \pm 38.7\end{array}$ \\
\hline 2 & 14.5 & 17 & 0.43 & 0.32 & 0.89 & 1.230 & 7.55 & $\begin{array}{l}62-190 \\
119.1 \pm 49.5\end{array}$ \\
\hline 3 & 18.0 & 19 & 0.27 & 1. 39 & 0.91 & 1.937 & 9.08 & $\begin{array}{l}70-181 \\
107.0 \pm 39.7\end{array}$ \\
\hline 4 & 17.5 & 13 & 0.37 & 0.95 & 0.73 & 1.442 & 3.55 & $\begin{array}{l}59-171 \\
109.6 \pm 40.6\end{array}$ \\
\hline 5 & 12.0 & 18 & 0.47 & -0.43 & 0.60 & 3.402 & 2.97 & $\begin{array}{l}67-241 \\
138.8 \pm 51.5\end{array}$ \\
\hline
\end{tabular}

a Heading symbols; $n=$ number of trials; $b_{1}=$ regression coefficient; $b_{0}=y$ intercept (in $\mathrm{ml}$ ); $r=$ correlation coefficient ; $s=$ standard error of estimate (in $\mathrm{ml}$ ); $t=$ Student's $t$ statistic.

$\mathrm{b}$ Range and mean $\pm 1 \mathrm{SD}$. 
tracked stroke volume, the volume of air calculated from the PNGG over the ejection period was consistently less than cardiac stroke volume.

\section{Discussion}

A search of the scientific literature back to the mid-1800's revealed numerous studies of the PNGG. However, most of these reports were simply descriptive and qualitative. Only one other group ${ }^{10,11)}$ has quantitatively related the PNCG to cardiodynamic parameters.

In one study, ${ }^{10)}$ Smith et al compared peak aortic blood flow in the baboon with the sum of amplitudes of the pneumocardiogram's systolic component. The sum of amplitudes consisted of the peak-to-peak HI and IJ amplitudes and the amplitude from baseline to the peak of the I wave (Fig. 1 ). They observed that the sum of amplitudes of the PNCG increased with increasing peak aortic blood flow. In a similar study, ${ }^{11}$ they observed that during ventricular systole the acceleration of tracheal air, determined from the time derivative of the PNCG, increased with increasing maximum acceleration of blood through the ascending aorta. It should be noted that Smith and coworkers described an outward flow of air during ventricular ejection, whereas the present authors observed an inward flow of air.

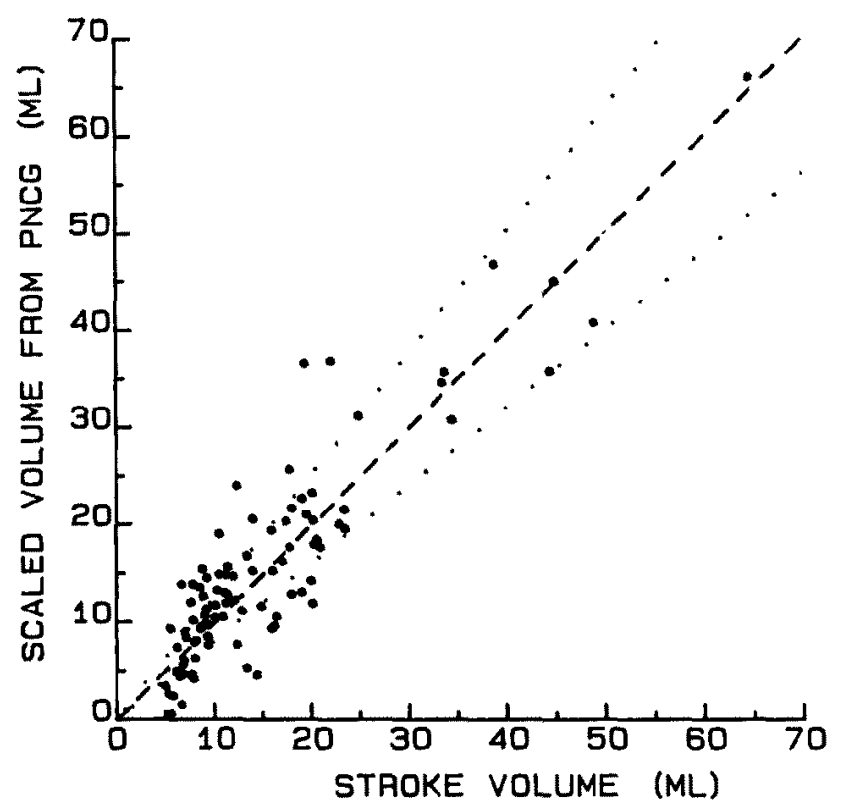

Fig. 4. Scaled pneumocardiographic volume versus stroke volume from 5 dogs. The dashed line is the regression line forced through the origin; the dotted lines represent $\pm 20 \%$. 


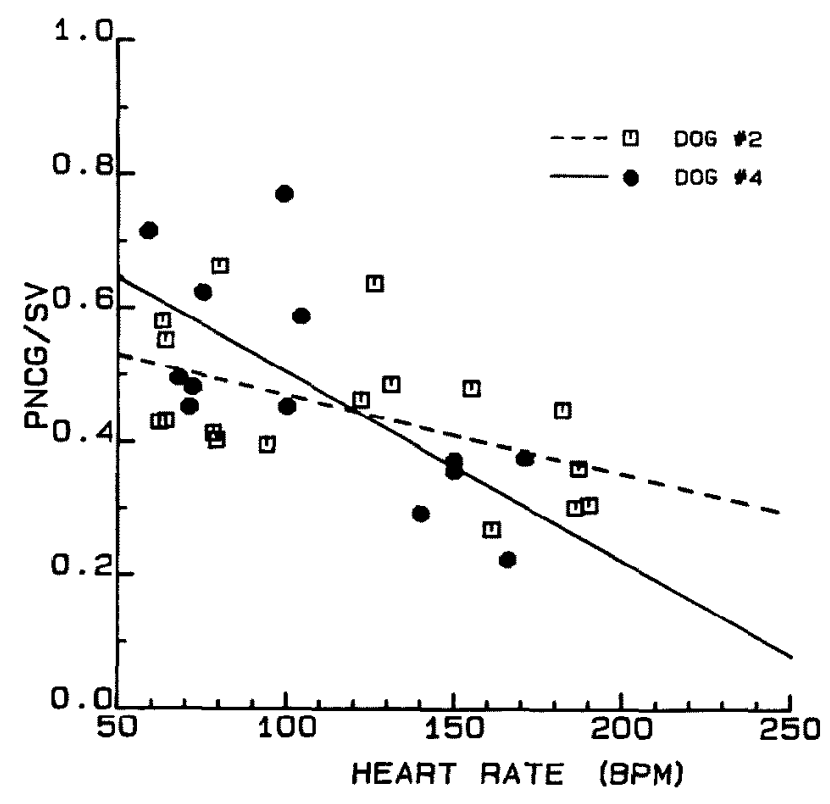

Fig. 5. PNCG/SV versus heart rate for dogs \#2 and \#4. Each line represents the linear regression line for the data from an individual dog.

A discussion of the mechanisms involved in generation of the cardiogenic air flow is beyond the scope of the present study. However, inferences can be made about the types of factors-either physiological or anatomicalwhich influence the PNCG-SV relationship. For example, these results suggest that heart rate-dependent as well as animal-dependent factors may confound the fundamental relationship.

Differences between animals are most apparent for dogs \#1 and \#2. Figs. 2 and 3 illustrate that for a stroke volume of $20 \mathrm{ml}$, the pneumocardiographic volume for $\operatorname{dog} \# 2$ is nearly twice that for $\operatorname{dog} \# 1$. To eliminate differences between animals, and therefore underscore the PNCG-SV relationship, the pneumocardiographic volume was multiplied by a scaling factor - the reciprocal of the linear regression coefficient (through the origin)calculated for each animal. The scaled data from all 5 animals are illustrated in Fig. 4. The regression coefficient is therefore 1.00 and the correlation coefficient is 0.90 . The dashed line in Fig. 4 represents the line of regression and the dotted lines represent the $\pm 20 \%$ range.

Heart rate-dependent differences in the PNCG-SV relationship were observed in nearly all the animals. For example, in dog \#1 (Fig. 2) the volume of air was disproportionately smaller for right atrial pacing at heart rates exceeding 150 beats/min (the diamonds at the lowest stroke volumes in Fig. 2). The most significant heart rate-dependence was observed for dogs \#2 
and \#4. Fig. 5 shows the relationship between heart rate and the ratio of pneumocardiographic volume to measured stroke volume (PNCG/SV) for these 2 animals. Linear regression of these data yielded correlation coefficients of -0.51 and -0.71 for dogs $\# 2$ and \#4, respectively. In general, PNCG/SV decreased with increasing heart rate (see Fig. 5); in particular, PNCG/SV was typically smallest for the high heart rates (above 150 beats/ min). Studies identifying the additional factors influencing the PNCG-SV relationship and their underlying mechanisms are indicated.

The data presented in this study may provide the basis for using the PNCG as a noninvasive indicator of ventricular stroke volume. The PNGG is recorded simply by connecting a pneumotachograph in series with the airway of the patient. The pneumotachograph offers little resistance to breathing and can be left in the breathing circuit. Whereas most invasive techniques yield average stroke volume, the PNCG provides a beat-by-beat indication of cardiac stroke volume, and it is well suited to assessing patient stability during closed-chest anesthesia and to monitoring post-operative recovery.

\section{REFERENCES}

1. Voit G: Ueber Druckschwankungen im Lungenraum in Folge der Herzbewegungen. $\mathrm{Z}$ Biol 1: 390, 1865

2. Landois L: Graphische Untersuchungen uber den Herzschlag im Normalen und Krankhaften Zustande. August Hirschwald, Berlin, p 1-47, 1876

3. Haycraft JB, Edie R: The cardiopneumatic movements. J Physiol (Lond) 12: 426, 1891

4. Geddes LA, Peery E, Steinberg R: Cardiac output using an electrically calibrated flowthrough conductivity cell. J Appl Physiol 37: 972, 1974

5. Worley DS, Grubbs DS, Geddes LA: Repeated cardiac output determination in the experimental animal. Physiologist 25: 175, 1982

6. Kinsman JM, Moore JW, Hamilton WF: Studies on the circulation. I. Injection method: physical and mathematical considerations. Am J Physiol 89: 322, 1929

7. Bourland JD: The Pneumocardiogram. A Noninvasive Respiratory Indicator of Cardiac Activity (Ph.D. dissertation), Houston, Texas, Baylor College of Medicine, 1974

8. Bourland JD, Wessale JL, Babbs CF, Milewski RC, Rockenhauser ME, Geddes LA, Hinds MH: Determination of cardiac stroke volume from the pneumocardiogram (abstr). Proceedings AAMI 15th Annual Meeting, Assoc Adv Med Instrum, Arlington, VA, p 17, 1980

9. Wessale JL, Bourland JD, Babbs CF, Rockenhauser ME, Milewski RG, Geddes LA: Cardiac stroke volume calculation from the pneumocardiogram. Med Electron (September, No 64), p 98-102, 1980

10. Smith NT, Van Citters RL, Verdouw PD: Relation between the pneumocardiogram and aortic flow in the baboon. Bibl Cardiol 24: 34, 1969

11. Smith NT, Reitan JA: The pneumocardiogram: a potential monitor for the operating room. Anesth Analg (Cleve) 49: 781, 1970

12. Neter J, Wasserman W: Applied Linear Statistical Models. Richard D Irwin, Inc, Homewood, IL, p 140-175, 1974 